\title{
Cloned cows turn back the cellular clock
}

Researchers at Advanced Cell Technology (ACT; Worcester, MA) have reported that cells from six cloned cows appear to be "younger" than either the donor cells from which they were cloned or comparable cells from control animals, suggesting cloning can reset, and possibly turn back, the clock on cellular aging (Science 288, 665-669). The authors claim the study has positive implications for therapeutic human stem cell applications, but other researchers question the commercial significance of the study.

In the ACT study, Robert Lanza and colleagues used nuclear transfer to clone cows from fetal-derived, senescent donor somatic cells. They found that the telomere lengths (which decrease with aging) of nucleated blood cells taken from the cloned calves were significantly longer than in cells from non-cloned, age-matched control animals. They also demonstrated between three- and fivefold higher levels of the agedependent marker gene EPC-1 mRNA (early population-doubling level complementary DNA-1) in cells from cloned calves compared to controls. According to the authors, " $[\mathrm{T}]$ he elongation of telomeres. . suggests that reconstructed bovine embryos contain a mechanism for telomere length regeneration," and that nuclear transfer can "restore somatic cells to a phenotypically youthful state."

In addition, another experiment showed that fibroblasts isolated from fetuses cloned from adult donor cells underwent more than 90 divisions in culture, compared to about 60 for cells from control animals-a greater than $50 \%$ extension of cell life span. "We documented that those cells, when they reached senescence, had shortened telomeres, and that after the cloning procedure, they were extended," says Lanza.

Although it remains to be seen whether increased life span at the cellular level will translate into prolonged survival of the cloned calves, the authors make lofty claims that the ability to extend the life span of specific cell types, such as liver and muscle cells and pancreatic islets, by an extra 30 divisions "would lead to a billionfold increase in the number of replacement cells generated for tissue engineering and transplantation therapies."

Following the cloning of Dolly the sheep from an adult quiescent mammary cell in 1997 (Nature, 385, 810), the ques-

Vicki Glaser is a freelance writer working in Allentown, PA.

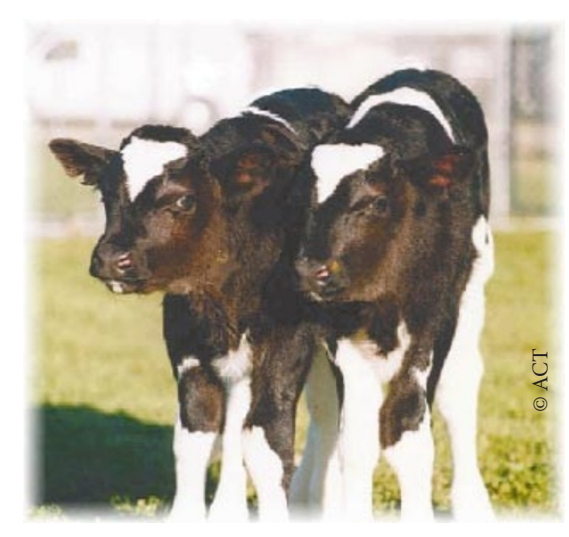

ACT researchers claim the cloned-cow study has positive implications for therapeutic human stem cell applications.

tion of premature aging of cloned tissues and cells arose when it appeared that Dolly's cells were "older" than she was, and closer in age to the donor cell from which she was cloned. "Hopefully, we have put that issue to rest," says Lanza. "The reason this paper is so important is that as you generate new tissues and organs using tissue engineering, this would suggest that those tissues and organs would at least live as long if not outlive the normal life span of the patient."

\section{From a scientific point of view, it's fascinating, but it's inconclusive as far as trying to sell this as an alternative way of producing tissues for human medical uses.}

"This is a very thorough study," comments Bob Wall, a scientist in the Gene Evaluation and Mapping Laboratory of the USDA Agricultural Research Service (Bethesda, MD). "From a scientific point of view, I think it's fascinating, but it's inconclusive as far as trying to sell this as an alternative way of producing tissues for human medical uses." He says the results need to be repeated in other species and by other laboratories, before one can begin to postulate on their therapeutic implications. Furthermore, Wall maintains that it is not yet clear whether lengthening telomeres is sufficient to extend life: "It is almost impossible to accept the concept that telomeres are the driving force in aging."
John Clark and Ian Wilmut of the Roslin Institute (Roslin, UK), where Dolly was cloned, and Carl Harley of Geron Corp. (Menlo Park, CA), which has licensed Roslin's nuclear transfer technology, also question the conclusions of the study. On page 599 of this issue, they suggest that differences in the donor cells or cloning technique used by ACT could account for the differences in telomere length between the calves and Dolly, and for the prolonged life span of cells derived from the cloned calves. They propose that the low cloning efficiency $(<0.5 \%$ overall cloning efficiency) achieved in the ACT study could allow for "selection of rare events" that could have given rise to donor cells that were not in fact senescent. They go on to speculate that telomere length or the rate of telomere loss could have been a factor in this selectionperhaps donor cells with "more robust telomeres" produced more surviving clones.

As to whether nuclear transfer could be used to return cells to a youthful state and produce billions of cells for human cell transplantation, Clarke and Wilmut say: "This would depend in the first instance upon the effect being observed in human cells and, to be practical, the efficiency of the process would need to be dramatically improved." They point out that proliferative capacity of cells can be enhanced by the simple introduction of telomerase, and they believe that therapies that require life span extension of human somatic cells "will come from the controlled expression of telomerase in culture, not from the manipulation of cloned animals." (Geron's research focuses on the use of telomerase to modulate cell senescence.)

In a written statement, PPL Therapeutics (Edinburgh, UK), which has licensed the Roslin technology for use in producing pharmaceuticals in transgenic animals, cautions that comparisons between the ACT data and the Dolly findings should be "made with some care" because Dolly was cloned from an adult cell, whereas the calves were cloned from fetal cells. "When we examined telomere length in sheep generated from fetal fibroblast cell nuclei, we found it to be indistinguishable from age-matched controls," the company says. The results are published in Cloning (1, 119-125, 1999).

The take-home lesson from the ACT paper, says Wall, is that ACT came up with different results than were seen in Dolly. "Somebody needs to ask the question why."

Vicki Glaser 